\title{
Optimal control of fed-batch fermentation involving multiple feeds using Differential Evolution
}

\author{
Mangesh D. Kapadi, Ravindra D. Gudi* \\ Department of Chemical Engineering, Indian Institute of Technology Bombay, Powai, Mumbai 400076, India
}

\begin{abstract}
Differential Evolution (DE), an exceptionally simple and robust evolutionary algorithm with Lagrangian like method, was used for solving optimal control and parameter selection problems of fed-batch fermentation involving general constraints on state variables. These infinite dimensional optimization problems were approximated into the finite dimensional optimization problems by control vector parameterization. Integration of the dynamic penalty functions was used to ensure the feasible solution of these dynamic optimization problems. State and end-point constraints were included in the formulation to reflect the operating objectives. The optimization strategy was able to accommodate these constraints in a relatively simple manner. The concept of non-uniform discretization in control vector parameterization was evaluated and shown to give superior results. Simple representative problems as well as a complex, multiple feed problem of simultaneous saccharification and fermentation (SSF) has been considered here to demonstrate the validity of the proposed methodology. The proposed methodology yielded an increase in productivity of approximately $20 \%$ in the case studies considered here.
\end{abstract}

Keywords: Differential Evolution; Fermentation; Optimization

\section{Introduction}

With a shift in focus towards the production of high value, low volume products, a number of chemical and biochemical processes operate in fed-batch mode. In exothermic batch reactors, some reactants are typically added in a fed- or semi-batch fashion to ensure proper temperature control. A large number of chemical processes, for example, fermentation processes, operate in fed-batch mode to avoid phenomena such as substrate or product inhibition and to achieve high productivities. The optimization of such processes requires solution of optimal control problems, that results in nutrient or promoter addition policies over the batch so that the desired objectives are met. The design of such control strategies which maximize or minimize a given performance index has been the focus of research work under the heading of optimal control. An excellent review of optimal control problems in batch distillation is provided in Diwekar [5].
Determination of optimal control policies in such semi-/fed-batch reactors typically involves the solution of non-linear optimization problems. Of critical importance is the form in which the control variable (typically nutrient or promoter addition) appears in the dynamic balance equations. In typical applications such as fed-batch fermentation, the control variable appears linearly in the dynamic balance and results in a relatively difficult problem formulation, termed as singular control problem [9]. Such problems are difficult to solve when the dynamic balance equations that describe the system increase in number, and the formulation becomes even more complex when one or more state constraints bound the problem. There are a number of alternate methods that convert this problem to result into a non-singular problem which could be easily solved [8]. In these methods, the transformation of the problem or the choice of new control variables is a critical step to achieve non-singular problem formulations.

Typically a fed-batch process could have more than one control variable that needs to be optimized. This leads to a fairly complex optimization problem when these control variables appear linearly in the dynamic balance equations. The transformation from singular to non-singular 
formulation as proposed by Modak and Lim [8] is also not straightforward for such fed-batch systems involving more than one control variables and physical constraints on state variables. This is because such simplifying transformations may not exist for the latter. The choice of alternate control variables may also be restricted due to the nature of the problem.

Of recent interest is the class of stochastic optimization methods that are becoming increasingly popular to solve such complex optimization problems involving discontinuities and other complex relationships. Differential Evolution (DE) as developed by Storn and Price [14] is a superior population based stochastic optimization method. The Differential Evolution algorithm operates on a population of potential solutions by applying the principle of survival of the fittest to produce an optimal solution. DE, a very simple computational algorithm, with only a few parameters required to be set by a user has proven to be a promising candidate to minimize real valued, multimodal objective functions [10]. In $\mathrm{DE}$, the fitness of an offspring competes one-to-one with that of the corresponding parent. This one-to-one competition, which is different from other evolutionary algorithms, gives rise to a fast convergence rate. Although this faster convergence leads to a higher probability of obtaining a local optimum, this drawback could be overcome by using a larger population size [10]. The Differential Evolution algorithm, which is a parallel direct search method, has been used in the recent past, to solve many practical optimization problems involving general constraints. Lee et al. [6] have demonstrated the results obtained by dynamic optimization of a continuous polymer reactor using a modified Differential Evolution. Babu and Sastry [2] have used DE for the estimation of effective heat transfer parameters in tricklebed reactors using radial temperature profile measurements fermentation process. The main advantage of using DE with augmented Lagrangian including penalty methods for fed-batch fermentation is that substrate feed rates can be used as control variables though there are several state inequality/equality constraints along with the bounds on the substrate feed rates. An optimal feed policy problem of a fed-batch fermentation process has been solved using a hybrid version of DE by Chiou and Wang [4]. Wang et al. [17] have successfully applied hybrid Differential Evolution to estimate the kinetic model parameters of batch fermentation process.

In this work, we analyze the computational aspects of Differential Evolution towards solving more complex dynamic optimization problems. Firstly, we extend the basic formulation of Chiou and Wang [4] for systems involving multiple feeds (and hence multiple control variables). Secondly, the key concept proposed by Chiou and Wang [4] during problem formulation, is the control vector parameterization over the batch duration. In control vector parameterization, the overall time behaviour of the control variable is partitioned over several smaller intervals of time duration, and the values that the control variable would take over each of these time intervals, are calculated via optimization. Chiou and Wang [4] have proposed that the time durations over which the control variable is parameterized, should be of equal length and have therefore called their approach as uniform parameterization. Here, we explore the possibility of non-uniform parameterization, i.e. we assume that the time duration of each of the smaller intervals need not be equal. We demonstrate that the superiority of the resulting formulation over the uniform parameterization case. We also additionally extend their formulation to accommodate both, path and end-point constraints on the state variables so as to more realistically define the problem in fed-batch reactors. It is demonstrated that Differential Evolution algorithm is able to successfully accommodate these constraints and yield superior results for the constrained dynamic optimization problem. As a case study, we consider the optimal control of simultaneous saccharification and fermentation (SSF) process that involves the addition of starch and glucose towards optimizing lactic acid product and demonstrate the utility of the algorithm towards obtaining higher productivities and improved operation.

This paper is organized as follows. In Section 2, we formulate the general optimal control problem for applications involving multiple feeds, path and end-point constraints and control vector parameterization. Section 3 discusses the application of the non-uniform control vector parameterization and multiple feeds to the problem considered by Chiou and Wang [4] and demonstrates the superiority of the proposed method. In Section 4, we consider the application of the proposed methodology to the optimization of the SSF process. Section 5 provides a summary of the key results derived from this work.

\section{Problem formulation}

Consider that the dynamic balance equations describing the evolution of each of the states (concentration of species, temperature, pressure, etc.) can be written for the fed-batch application as

$\underline{\dot{x}}=f(\underline{x}(t), \underline{u}(t), t, \underline{\theta})$

In the above equation, $\underline{x}$ is the vector of system states, $\underline{u}$ is the vector of control variables that needs to be optimized and $\underline{\theta}$ is the vector of system parameters. The initial conditions of the dynamic system are specified as

$\underline{x}(0)=\underline{x}_{0}$

These initial conditions are either known/set a priori or could themselves be considered as decision parameters in the optimization problem.

A general criterion for the optimization of the fed-batch process is typically expressed in terms of indices defining the end concentration of a product and/or the dynamic state values themselves and can be suitably expressed as [11]

$J=G\left(\underline{x}\left(t_{\mathrm{f}}\right), t_{\mathrm{f}}\right)+\int_{t_{0}}^{t_{\mathrm{f}}} F(\underline{x}(t), \underline{u}(t), t, \underline{\theta}) \mathrm{d} t$ 
A number of constraints bind the solution space of the above problem. These typically reflect the practical objectives (i.e. those on state variables) and operating constraints (those on the control variables), and can be written as

$\underline{x}_{\min } \leq \underline{x} \leq \underline{x}_{\max }$

$\underline{u}_{\min } \leq \underline{u} \leq \underline{u}_{\max }$

In the above equations, the state constraints are essentially path constraints, i.e. their values during the progress of the batch are constrained to lie within the upper and lower limits. Typically examples are the nutrient levels in fermenter should not exceed an inhibitory value. Similarly, practical constraints on the flow rates achievable through nutrient additions pumps may get translated into control vector constraints. An additional constraint typically implemented for the downstream processing and separation viewpoint is that the end concentration of a particular reactant needs to be small and below given threshold value. The constraint on the final volume of the semi-batch reactor also falls into this category.

$\left(\underline{x}\left(t_{\mathrm{f}}\right)-\underline{x}_{\mathrm{f}}\right) \leq 0$

Additionally, some equality or inequality constraints on state variables should be considered to obtain realistic solutions. These constraints are typically the constraints due to the stoichiometry of the process.

An excellent summary of methods based on maximum principle and gradient based methods for both singular and non-singular problems has been presented in Ray [11]. However, as mentioned earlier, the solution procedure becomes typically quite complex when the size of the control vector $\underline{u}$ increases. Also, for singular problems simplifying transformations to convert the problem into a non-singular form are not easy to obtain.

A particularly attractive technique variously proposed $[3,11]$ is the parameterization of the control vector $\underline{u}$ into a number of intervals so to result in an expression as

$u_{i k}(t)=\sum_{j=1}^{8} a_{i j k} \phi_{i j}(t)$

where $i=1, \ldots$, number of intervals; $k=1, \ldots$, number of control variables. In the above equation, $\phi_{i j}(t)$ are known trial functions that need to be chosen, $u_{i k}(t)$ is the value of the $k$ th control variable in $i$ th interval of the horizon and $a_{i j k}$ are the coefficients that need to be determined from optimization. A number of trial functions based on the use of different basis functions have been used in earlier works [3]; however, the choice of trial functions $\phi_{i j}(t)$ affects the evaluation of the exact optimization and needs to be carefully chosen. In this work, we assume that each control variable in the control vector $\underline{u}(t)$ is approximated by a piecewise constant value in a particular interval $i$. A study assessing the choice of various other trial factors is presently in progress. Furthermore, while the number of such intervals has been assumed to be fixed as in Chiou and Wang [4], the time duration of each interval is allowed to vary in this work, to result in a non-uniform parameterization of the control vector.

The decision parameters, $z=\left[z_{1}, \ldots, z_{n}\right]$ for the above optimization problem can be written to be equal $((k+1) \mathrm{Nu}+$ $P_{\mathrm{s}}$ ), where Nu indicates the number of intervals of the horizon and $P_{\mathrm{s}}$ is the number of system parameters needed to be optimized. Here, we propose to solve the optimization problem defined in Eqs. (1)-(7) in the space of the above decision parameters. In particular, we propose to use the Differential Evolution algorithm with Lagrange like method including penalty functions. In this algorithm, DE utilizes $N_{\mathrm{p}}$ vectors of decision parameters $z$ in the optimization problem, i.e. $z_{i}^{G}, i=1,2, \ldots, N_{\mathrm{p}}$ as a population for each generation $G$. The initial population is randomly selected and attempts to cover the entire solution space uniformly [13].

$z_{i}^{0}=z_{\min }+\rho_{i}\left(z_{\max }-z_{\min }\right), \quad i=1,2, \ldots, N_{\mathrm{p}}$

where $z_{\min }$ and $z_{\max }$ are the lower and upper bounds of the decision parameters, respectively, and $\rho_{i}$ denotes the uniformly distributed random numbers. There are three main operations in DE, viz. mutation, crossover and evaluation. The mutation operation at the $(G-1)$ th generation begins by randomly selecting two or four population individuals which are then combined to form a difference vector. The weighted difference vector is added to third randomly selected individual or best performing individual to have perturbed individual $\hat{z}_{i}^{G}$ [13].

$\hat{z}_{i}^{G}=z_{\mathrm{p}}^{G-1}+F\left(z_{j}^{G-1}-z_{k}^{G-1}\right)$

where $F \in(0,1.2]$ is a scaling factor. The randomly chosen integers $p, j$ and $k$ are chosen to be different from running index $i$. The resulting perturbed individual and target individual in current generation are selected by a binomial distribution to perform crossover operation to generate an offspring [15].

$z_{j i}^{G}= \begin{cases}z_{j i}^{G-1}, & \text { if a random number }>C_{\mathrm{R}} \\ \hat{z}_{j i}^{G-1}, & \text { otherwise }\end{cases}$

where $j=1, \ldots, n ; i=1, \ldots, N_{\mathrm{p}}$ and the crossover factor $C_{\mathrm{R}} \in[0,1]$ is to be set by user. In DE, the evaluation function of an offspring competes one to one [15] with that of its parent (target individual in current generation), i.e. the parent is replaced by its offspring in the next generation only if the objective function value of the offspring is lower than that of its parent.

$z_{i}^{G}=\arg \min \left\{J\left(z_{i}^{G-1}\right), J\left(z_{i}^{G}\right)\right\}, \quad i=1, \ldots, N_{\mathrm{p}}$

The best individual will be

$z_{b}^{G}=\arg \min \left\{J\left(z_{i}^{G}\right)\right\}, \quad i=1, \ldots, N_{\mathrm{p}}$

As the DE algorithm is for unconstrained minimization problems, methods based on dynamic penalty functions [7] 
are used to convert the constrained problem in unconstrained problem. In these methods, penalty terms associated with constraints are added to the objective function, i.e. penalty terms reflect the violation of the constraints and assign higher costs of the penalty function to the individuals that are far from the feasible region.

$J_{a}(z)=J(z)+(C+G)^{\alpha} \sum_{k=1}^{m} J_{k}^{\beta}(z)$

where $C, \alpha$ and $\beta$ are constants and $m$ represents total number of constraints. A reasonable choice for these parameters is $C=0.5, \alpha=\beta=2$. In the above equation, $J_{k}(z)(1 \leq$ $k \leq m$ ) represents a set of functions used to construct the penalty, where the function $J_{k}$ measures the violation of the $k$ th constraint. This method requires much small number of parameters (independent of the number of constraints). Also, the pressure on infeasible solutions increases due to the $(C+$ $G)^{\alpha}$ component of the penalty term. Hence any candidate individual that violates the constraints would inherit a worse fitness value and will find it difficult to survive in the next generations.

\section{Case study I: optimization studies of ethanol production}

In this section, we consider ethanol producing fermentation of Chiou and Wang [4] wherein the feedrate of glucose was optimized to maximize ethanol production. We illustrate the utility of the non-uniform control vector parameterization and the multiple feed formulation for the same problem. The dynamic mass balance equations and problem formulation has been taken from Chiou and Wang [4] and Wang and Shyu [16].
Table 1

Comparison of results obtained by non-uniform control vector parameterization and uniform control vector parameterization for ethanol production

\begin{tabular}{lll}
\hline Parameters & $\begin{array}{l}\text { Results with } \\
\text { non-uniform } \\
\text { control vector } \\
\text { parameterization }\end{array}$ & $\begin{array}{l}\text { Results with uniform } \\
\text { control vector } \\
\text { parameterization [4] }\end{array}$ \\
\hline End product $(\mathrm{kg})$ & 85.27 & 84.63 \\
Production rate $(\mathrm{kg} / \mathrm{h})$ & 4.794 & 4.675 \\
Fermentation time $(\mathrm{h})$ & 17.78 & 18.1 \\
Residual glucose $(\mathrm{g} / \mathrm{l})$ & 0.12 & 0.97 \\
\hline
\end{tabular}

\subsection{Non-uniform control vector parameterization}

The optimization problem for fed-batch fermentation of ethanol production by Zymomous mobilis was solved with uniform control vector parameterization (fixed time interval) in the work of Chiou and Wang [4]. In this work, the concept of variable time interval length discussed in Section 2 was applied to the same optimization problem. A variable length for each time interval

$\tau_{i}=t_{i}-t_{i-1}, \quad i=1, \ldots, \mathrm{Nu}$

is considered as a decision variable in the optimization problem. Here, $\tau_{i}, i=1, \ldots, \mathrm{Nu}$ are referred to as time interval size parameters. The sum of all time interval size parameters should be within the range of fermentation time. Hence, the constraints

$$
\begin{aligned}
& \sum_{i=1}^{\mathrm{Nu}} \tau_{i}-t_{\mathrm{f}, \max } \leq 0, \quad i=1, \ldots \mathrm{Nu} \\
& t_{\mathrm{f}, \min }-\sum_{i=1}^{\mathrm{Nu}} \tau_{i} \leq 0, \quad i=1, \ldots, \mathrm{Nu}
\end{aligned}
$$

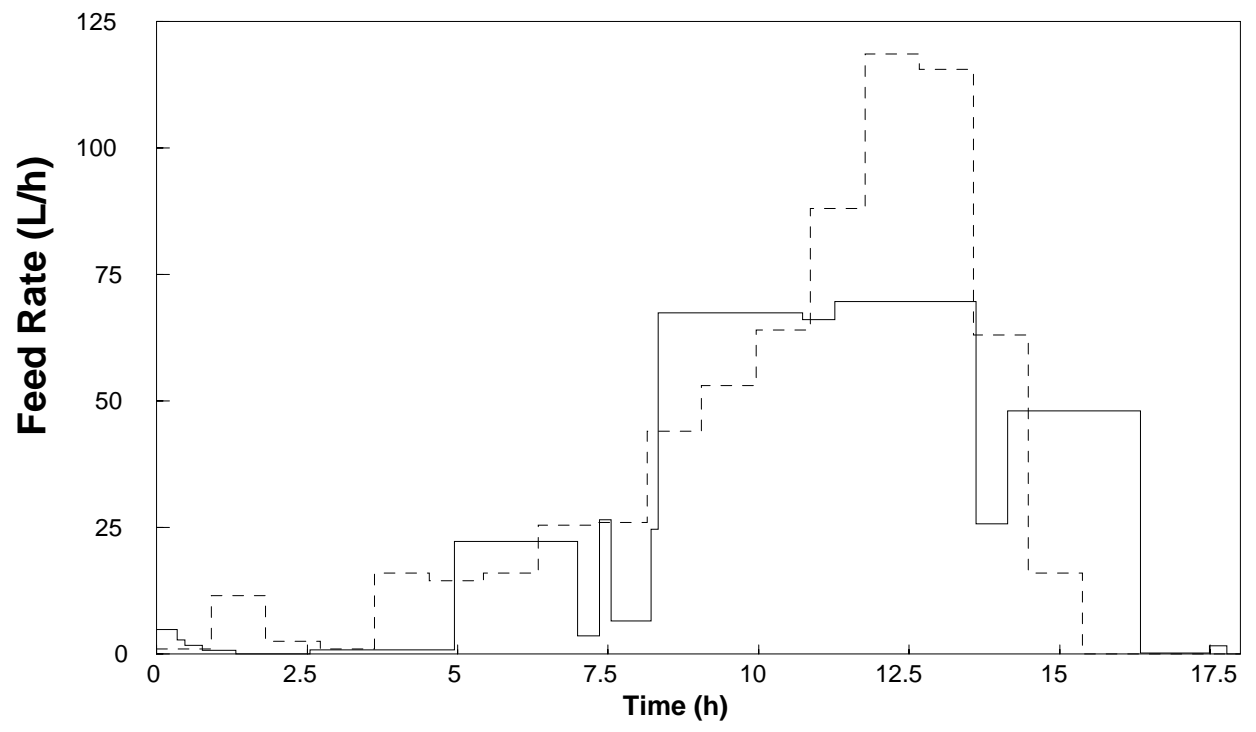

Fig. 1. Optimal feed rates for ethanol production; solid line for non-uniform control vector parameterization; dotted line for uniform control vector parameterization [4]. 


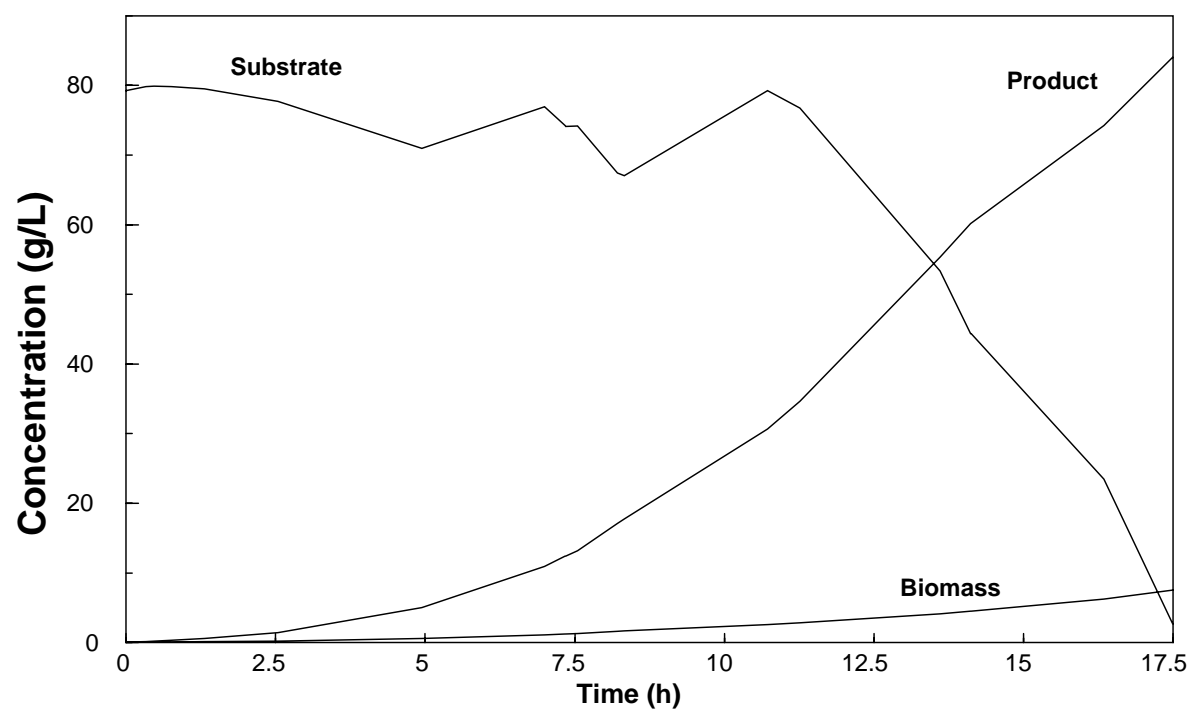

Fig. 2. Optimal concentration profiles with non-uniform control vector parameterization for ethanol production.

are included in the optimization problem to obtain realistic solution. To solve this problem, the feed rate is first discretized into a set of 20 piecewise constant control actions. The Differential Evolution with Lagrange like method including penalty functions is used to solve the resulting optimization problem with 43 decision parameters (20 constant feed flow rates, 20 time interval size parameters, $G_{\mathrm{F}}, G_{0}$ and $\left.V_{0}\right)$. The crossover factor in the DE algorithm is set to be 0.5. A population size of 90 and an initial penalty parameter of $10^{6}$ are used in the computation. The mutation factor $F_{\mathrm{m}}$ is taken as a random number in the interval $[0,1]$. The maximum feed flow rate and total working volume are considered as $F_{\max }=200 \mathrm{l} / \mathrm{h}$ and 10001 . The path constraints to ensure that the glucose concentration does not exceed $80 \mathrm{~g} / \mathrm{l}$ are imposed. Additionally, the final concentration of glucose is also constrained to be less than $1 \mathrm{~g} / \mathrm{l}$ to ensure complete consumption of glucose added.

The maximum ethanol product of $85.27 \mathrm{~kg}$ (equivalent to the maximum production rate of $4.794 \mathrm{~kg} / \mathrm{h}$ ) is obtained after 20,000 iterations. The optimal parameters for the concentration of glucose in feed, initial glucose concentration and initial volume obtained by this approach are $G_{\mathrm{F}}=249.6 \mathrm{~g} / \mathrm{l}$, $G_{0}=79.3 \mathrm{~g} / 1, V_{0}=399.81$. The sum of all time intervals is found to be $17.78 \mathrm{~h}$. The residual glucose at the final time is $0.12 \mathrm{~g} / \mathrm{l}$. The optimal feed concentration of glucose, optimal initial concentration of glucose and the optimal initial working volume are very close to the upper bound of the solution space. The optimal feed flow rates and optimal concentration profiles for biomass, glucose and ethanol are shown in

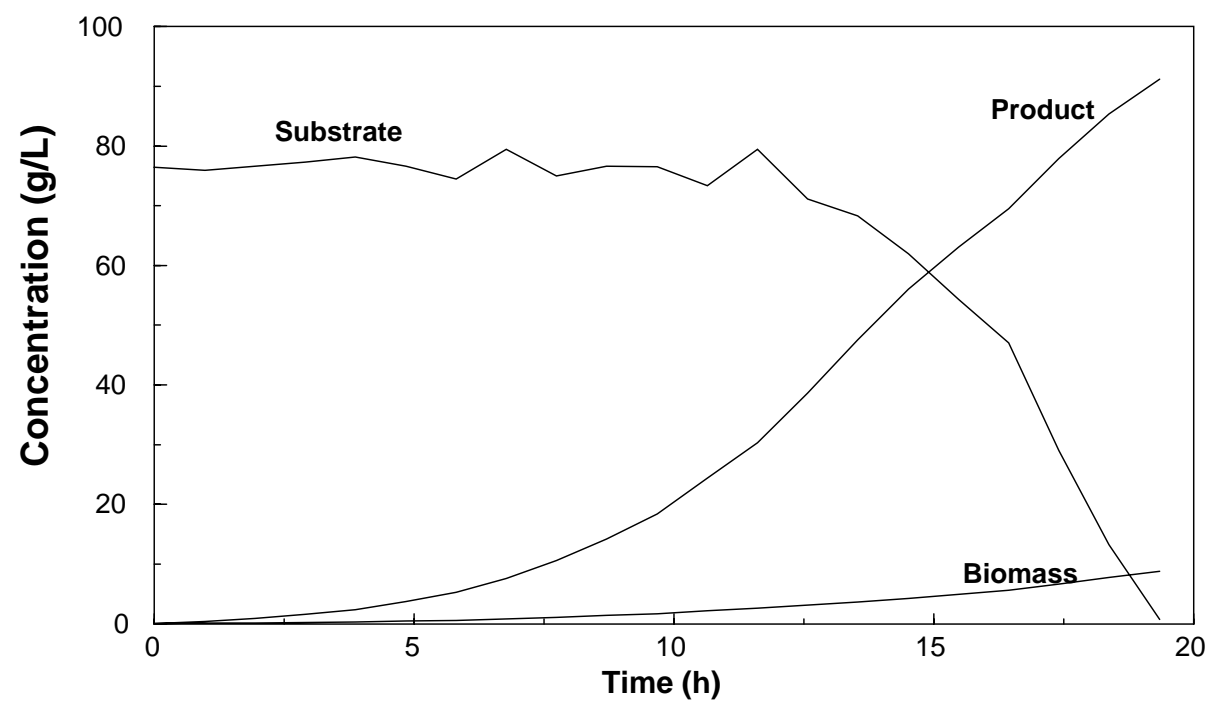

Fig. 3. Optimal concentration profiles for ethanol production with multiple feeds. 
Figs. 1 and 2. It is interesting to note that an improvement is obtained in the end ethanol concentration and in production rate due to non-uniform control vector parameterization. A comparison of results obtained using non-uniform control vector parameterization with those reported in Chiou and Wang [4] is shown in Table 1. It is seen from Table 1 that a higher final concentration of ethanol is obtained with higher production rate resulting in smaller fermentation time when compared with the results of Chiou and Wang [4]. It is also seen from Table 1, that the end-point constraint on the glucose is met. This ensures that the conversion of glucose is complete and there is no residual glucose in the final broth. In industrial fermentation, this result has an important impact that separation costs would be low in the downstream processing. It is also seen that the maximum feed flow rate of $70 \mathrm{l} / \mathrm{h}$ with the formulation proposed here is less than that of $1181 / \mathrm{h}$ (approximately) as reported by Chiou and Wang [4]. This is because the optimizer selects larger time intervals in the middle of fermentation when the substrate concentrations are not inhibitory. Thus, non-uniform control vector parameterization and end-point constraint can be accommodated to give superior results in terms of fermentation productivity.

\subsection{Extension to multiple feeds}

In this section, we consider the extension of the system considered in the earlier sub-section, to evaluate optimized profiles for two feed case. To enable this, we fictitiously divide the overall glucose addition into two streams, the first is a relatively concentrated (in glucose) feed $F_{1}$ and the other is a relatively dilute feed $F_{2}$. Then the dynamic mass
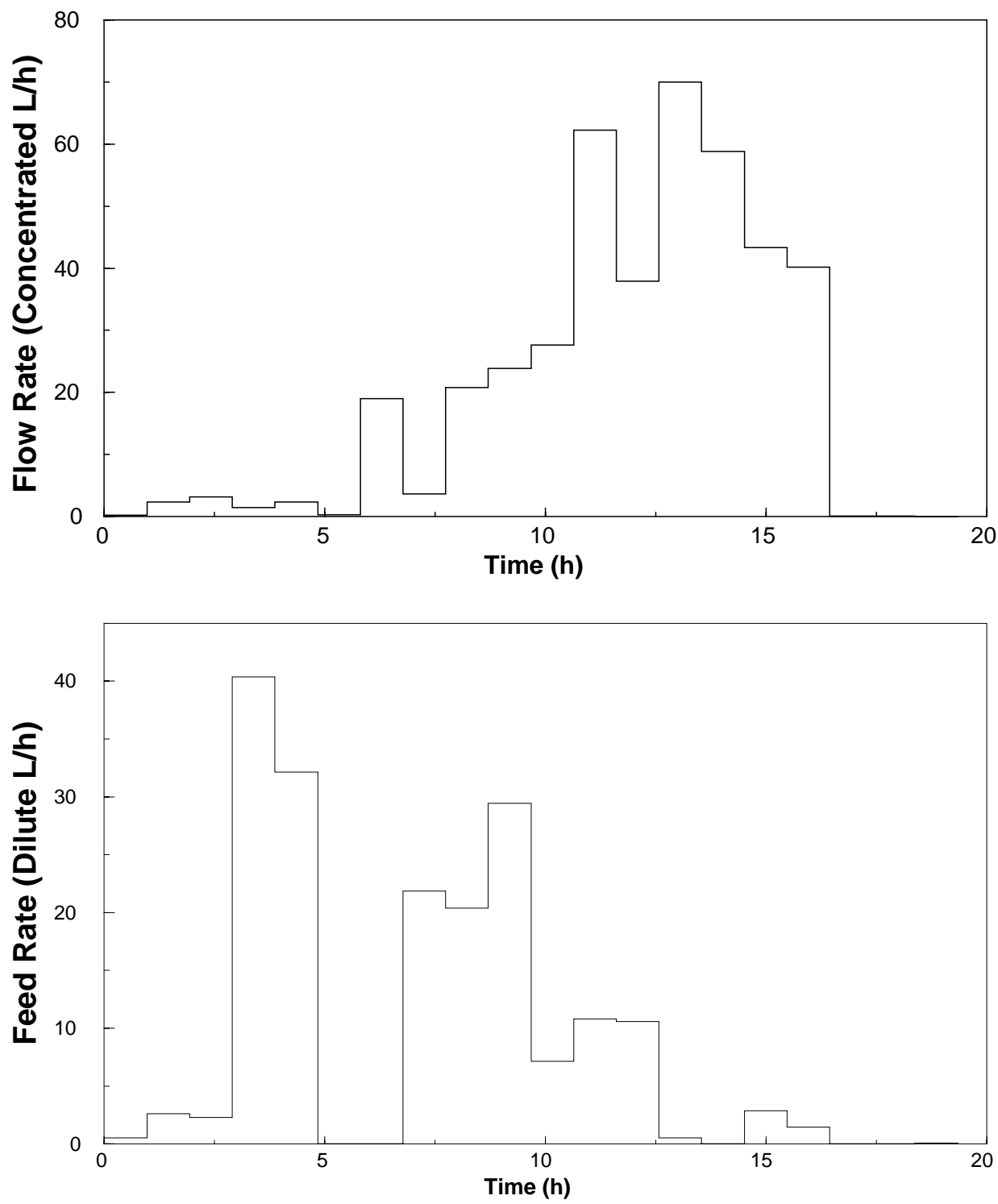

Fig. 4. Optimal feed flow rates for ethanol production with multiple feeds. 
balance Eq. (4) can be written as

$$
\begin{aligned}
\frac{\mathrm{d} X}{\mathrm{~d} t} & =\mu X-\frac{F_{1}+F_{2}}{V} X \\
\frac{\mathrm{d} G}{\mathrm{~d} t} & =-\frac{q_{\mathrm{p}}}{y_{\mathrm{p} / \mathrm{s}}} X+\frac{F_{1}}{V}\left(G_{F_{1}}-G\right)+\frac{F_{2}}{V}\left(G_{F_{2}}-G\right) \\
\frac{\mathrm{d} P}{\mathrm{~d} t} & =q_{\mathrm{p}} X-\frac{F_{1}+F_{2}}{V} P \\
\frac{\mathrm{d} V}{\mathrm{~d} t} & =F_{1}+F_{2}
\end{aligned}
$$

where $G_{F_{1}}$ and $G_{F_{2}}$ are the concentration of glucose in $F_{1}$ and $F_{2}$, respectively. The kinetic model of the specific growth rate $\mu$ and product formation $q_{\mathrm{p}}$ are taken to be the same as given in Chiou and Wang [4]. To take into account the effect of multiple feeds addition, the constraint on the specific yield of ethanol is modified as,

$$
\begin{aligned}
& \frac{P\left(t_{i}\right) V\left(t_{i}\right)-P(0) V(0)}{F_{\text {total }_{1}}\left(t_{i}\right) G_{F_{1}}+F_{\text {total }_{2}}\left(t_{i}\right) G_{F_{2}}+V(0) G(0)-V\left(t_{i}\right) G\left(t_{i}\right)} \\
& -Y_{\mathrm{p} / \mathrm{s}} \leq 0, \quad i=1, \ldots, \mathrm{Nu}
\end{aligned}
$$

where

$$
\begin{aligned}
& F_{\text {total }_{1}}\left(t_{i}\right)=F_{\text {total }_{1}}\left(t_{i-1}\right)+\tau_{i} F_{1}\left(t_{i}\right), \quad i=1, \ldots, \mathrm{Nu} \\
& F_{\text {total }_{2}}\left(t_{i}\right)=F_{\text {total }_{2}}\left(t_{i-1}\right)+\tau_{i} F_{2}\left(t_{i}\right), \quad i=1, \ldots, \mathrm{Nu}
\end{aligned}
$$

Differential Evolution method with a population size $N_{\mathrm{p}}=90$ is used to solve this optimization problem with 45 decision variables (20 constant flow rates for feed $F_{1}$ and $F_{2}$ each, $G_{F_{1}}, G_{F_{2}}, G_{0}, V_{0}$ and $\left.t_{\mathrm{f}}\right)$. All the other parameters are set to be same as mentioned in the earlier subsection. The upper and lower bounds for $G_{F_{1}}$ and $G_{F_{2}}$ are 350 and $250 \mathrm{~g} / \mathrm{l}$, and 200 and $100 \mathrm{~g} / \mathrm{l}$, respectively.

The maximum ethanol of $91.24 \mathrm{~kg}$ (equivalent to the maximum production rate of $4.719 \mathrm{~kg} / \mathrm{h}$ ) is obtained after 30,000 iterations. The optimal system parameters obtained for this problem are $G_{F_{1}}=350 \mathrm{~g} / 1, G_{F_{2}}=100 \mathrm{~g} / 1, G_{0}=76.46 \mathrm{~g} / \mathrm{l}$, $V_{0}=399.821$ and $t_{\mathrm{f}}=19.33 \mathrm{~h}$. The residual glucose at final time is $0.76 \mathrm{~g} / \mathrm{l}$. It is important to note that the concentration of substrate in feed $F_{1}$ is at its upper bound while that in feed $F_{2}$ is at its lower bound of the solution space. This indicates that the system prefers one concentrated and the other dilute source for glucose. The sum of all constraint violations equal to zero indicates that the solution so obtained is completely feasible. The optimal concentration profiles and optimal feed flow rates are shown in Figs. 3 and 4, respectively. From Fig. 4, it is clear that in the initial part of the fermentation, the addition of dilute source of glucose is preferred to keep the substrate concentration in fermentation broth below the inhibitory value of $80 \mathrm{~g} / \mathrm{l}$. Addition of glucose (concentrated or dilute) has a moderate dilution effect on the ethanol concentration. It is therefore interesting to note that the optimizer predicts addition of concentrated glucose, in the middle of the fermentation time, to minimize the dilution effect. Towards the end of fermentation, the optimizer predicts no addition of glucose source (dilute or concentrated) to keep the concentration of glucose at the end less than the residual limit. As a result, the maximum ethanol product obtained using multiple feeds is $7.8 \%$ higher than that obtained with single feed.

\section{Case study II: dynamic optimization of SSF process}

In this section, we illustrate the utility of the proposed method for dynamic optimization of simultaneous saccharification and fermentation of starch to lactic acid. The objective of the work is to maximize the final lactic acid concentration, productivity (i.e. amount of lactic acid obtained per unit volume per unit time) and yield with effective use of substrates (starch and glucose) against glucose and lactic acid inhibitions [12].

\subsection{Single feed optimization of SSF process}

The model equations for saccharification and fermentation processes are combined to form the model equations for simultaneous saccharification and fermentation process [12]. If it is assumed that only a single feed is being added which contains starch with some percentage of glucose (produced during autoclaving of starch), then the dynamic mass balance equations for SSF process [12] are

$\frac{\mathrm{d} X}{\mathrm{~d} t}=-\frac{F}{V} X+\mu X$

$\frac{\mathrm{d} S}{\mathrm{~d} t}=\frac{F}{V}\left(S_{\mathrm{F}}-S\right)-\frac{R_{\mathrm{G}}}{1.11}$

$\frac{\mathrm{d} G}{\mathrm{~d} t}=\frac{F}{V}\left(G_{\mathrm{F}}-G\right)+\left(R_{\mathrm{G}}-(a \mu X+b X)\right)$

$\frac{\mathrm{d} P}{\mathrm{~d} t}=-\frac{F}{V} P+(\alpha \mu X+\beta X)$

$\frac{\mathrm{d} V}{\mathrm{~d} t}=F$

where $S_{\mathrm{F}}$ and $G_{\mathrm{F}}$ are the concentration of starch and glucose in feed. The kinetic model of the specific growth rate $\mu$ and the rate of saccharification $R_{\mathrm{G}}$ in SSF process [1] are expressed as

$\mu=\mu_{\mathrm{m}} \mathrm{e}^{-K_{\mathrm{L}} P}\left(\frac{G}{K_{\mathrm{s}}+G+G^{2} / K_{\mathrm{I}}}\right)$

$R_{\mathrm{G}}=V_{\mathrm{m}}^{0} \mathrm{e}^{-k P^{n}} \frac{S}{K_{\mathrm{m}}\left(1+G / K_{\mathrm{G}}\right)+S}$

The values of the total 13 parameters at initial starch concentration of $30 \mathrm{~g} / \mathrm{l}$, at a temperature of $45^{\circ} \mathrm{C}$ and a $\mathrm{pH}$ of 5.6 are listed in Table 2 . In order to obtain realistic solution, the total volume of the fermenter is bounded by

$g_{1}=V(t)-V_{\mathrm{f}} \leq 0$ 
Table 2

Model parameters for lactic acid production by simultaneous saccharification and fermentation process [12]

\begin{tabular}{lclc}
\hline Parameter & Value & Parameter & Value \\
\hline$a$ & 6.9 & $b$ & 0.023 \\
$k$ & 0.02 & $n$ & 0.4 \\
$K_{\mathrm{m}}$ & 95.6 & $K_{\mathrm{G}}$ & 33.0 \\
$K_{\mathrm{S}}$ & 6.65 & $K_{\mathrm{L}}$ & 0.0037 \\
$K_{\mathrm{I}}$ & 104.5 & $V_{\mathrm{m}}^{0}$ & 68 \\
$\alpha$ & 9.2 & $\beta$ & 0.073 \\
$\mu_{\mathrm{m}}$ & 0.25 & & \\
\hline
\end{tabular}

The concentration of glucose and starch must be positive at all times.

$g_{2}=-S(t) \leq 0$

$g_{3}=-G(t) \leq 0$

Additionally, the final concentrations of starch and glucose are constrained to ensure complete consumption, i.e. the following constraints are included to reduce the downstream processing cost of lactic acid.

$g_{4}=S\left(t_{\mathrm{f}}\right)-S_{\mathrm{r}} \leq 0$

$g_{5}=G\left(t_{\mathrm{f}}\right)-G_{\mathrm{r}} \leq 0$

In order to avoid large variation in control action, the flow rates are constrained as

$g_{5+l}=|F(l+1)-F(l)| \leq 0.25 F_{\max }, \quad l=1, \ldots, \mathrm{Nu}-1$

The objective of the optimization problem is to maximize the lactic acid production rate with effective use of the substrate source.

$\max _{F(t)} J=\frac{p\left(t_{\mathrm{f}}\right) V\left(t_{\mathrm{f}}\right)}{t_{\mathrm{f}}}$

where the decision variables $F(t)$ are bounded as

$F_{\min } \leq F(t) \leq F_{\max }$

The penalty function method is used to transform the constrained optimization problem into a unconstrained problem. Hence, the augmented objective function is given as

$$
\begin{aligned}
\max _{F(t), t_{\mathrm{f}}} J_{a}= & J+\sum_{k=1}^{5} \int_{0}^{t_{\mathrm{f}}} \alpha_{k}(t)\left\langle g_{k}(x(t), F)\right\rangle_{+}^{2} \\
& +\mathrm{d} t+\sum_{k=1}^{\mathrm{Nu}-1} \alpha_{5+k}\left\langle g_{5+k}\right\rangle_{+}^{2}
\end{aligned}
$$

where $\alpha_{k}$ are positive penalty parameters and the bracket operator is defined as $\left\langle g_{k}\right\rangle_{+}=\max \left\{g_{k}, 0\right\}$. The state variable vector $x(t)$ is expressed as $x=[X, S, G, P, V]^{\mathrm{T}}$.

The Differential Evolution with Lagrange like method including dynamic penalty function is used to solve the optimization problem of lactic acid production. To solve this optimization problem the feed rate $F(t)$ is first approximated by 40 time partitions. The setting factors used for run are listed as follows. The population size of 75 , crossover factor $C_{\mathrm{R}}=0.5$ and the mutation scheme $\mathrm{DE} / \mathrm{best} / 2$ are used for the computational run. The mutation factor is taken as a random number in the interval $[0,1]$. The maximum iterations of 10,000 and an initial penalty parameter of $10^{6}$, are used in Differential Evolution algorithm to obtain minimum solution for the augmented Lagrange function. It is clear from the results of Roy et al. [12] that in order to build up high lactic acid concentration, it is first necessary to start with low substrate concentration and then feed should be added at much higher concentration. In this context, this optimal control problem is solved with a low initial starch concentration of $30 \mathrm{~g} / \mathrm{l}$. The feed contains starch at a concentration, $S_{\mathrm{F}}=200 \mathrm{~g} / \mathrm{l}$ with some glucose $\left(G_{\mathrm{F}}=\right.$ $15 \%$ of $S_{\mathrm{F}}$ ) available due to autoclaving. The concentration of glucose prior to start of SSF process due to autoclaving of initial starch present is found to be $4.1 \mathrm{~g} / \mathrm{l}$. The initial biomass concentration is taken as $0.5 \mathrm{~g} / \mathrm{l}$ and the initial and final working volume are 5 and 1001 , respectively. The residual limits on starch and glucose concentration are taken as 1.0 and $0.1 \mathrm{~g} / \mathrm{l}$. The fermentation time is decided to be $80 \mathrm{~h}$ while the upper bound for feed addition, $F_{\max }$ is $2.0 \mathrm{l} / \mathrm{h}$.

The maximum lactic acid concentration of $409.45 \mathrm{~g} / \mathrm{l}$, which is equivalent the production rate of $511.73 \mathrm{~g} / \mathrm{h}$ is obtained using proposed method. The residual concentration of starch and glucose obtained are 0.97 and $0.001 \mathrm{~g} / \mathrm{l}$, respectively. The sum of all constraint violation equal to zero indicates that the solution so obtained is completely feasible. The optimal feed flow rates and optimal concentration profiles for biomass, starch, glucose and lactic acid are shown in Fig. 5. The final lactic acid concentration and maximum lactic acid production rate obtained by proposed algorithm are greater than those obtained by Roy et al. [12] using Pontryagin's Maximum Principle with fermentation volume as control variable. From Fig. 5, it is clear that the end-point constraints on starch and glucose are met, i.e. there is no residual substrate in final fermentation broth. In industrial terms, it means the separation costs of lactic acid would be low in the downstream processing.

\subsubsection{Non-uniform control vector parameterization}

The dynamic optimization problem of simultaneous saccharification and fermentation of starch to lactic acid was solved with uniform control vector parameterization in earlier section. In this section, the concept of different time interval size parameters (i.e. non-uniform control vector parameterization) discussed in Section 3.1 is applied to the same optimization problem of SSF process. The proposed modifications discussed in Section 3.1, are made in the main optimization problem and it is solved twice, firstly taking the fermentation time as decision variable and secondly keeping it fixed as $80 \mathrm{~h}$. While solving the resulting optimization problem with $t_{\mathrm{f}}=80 \mathrm{~h}$, the $(\mathrm{Nu})$ th time interval size 

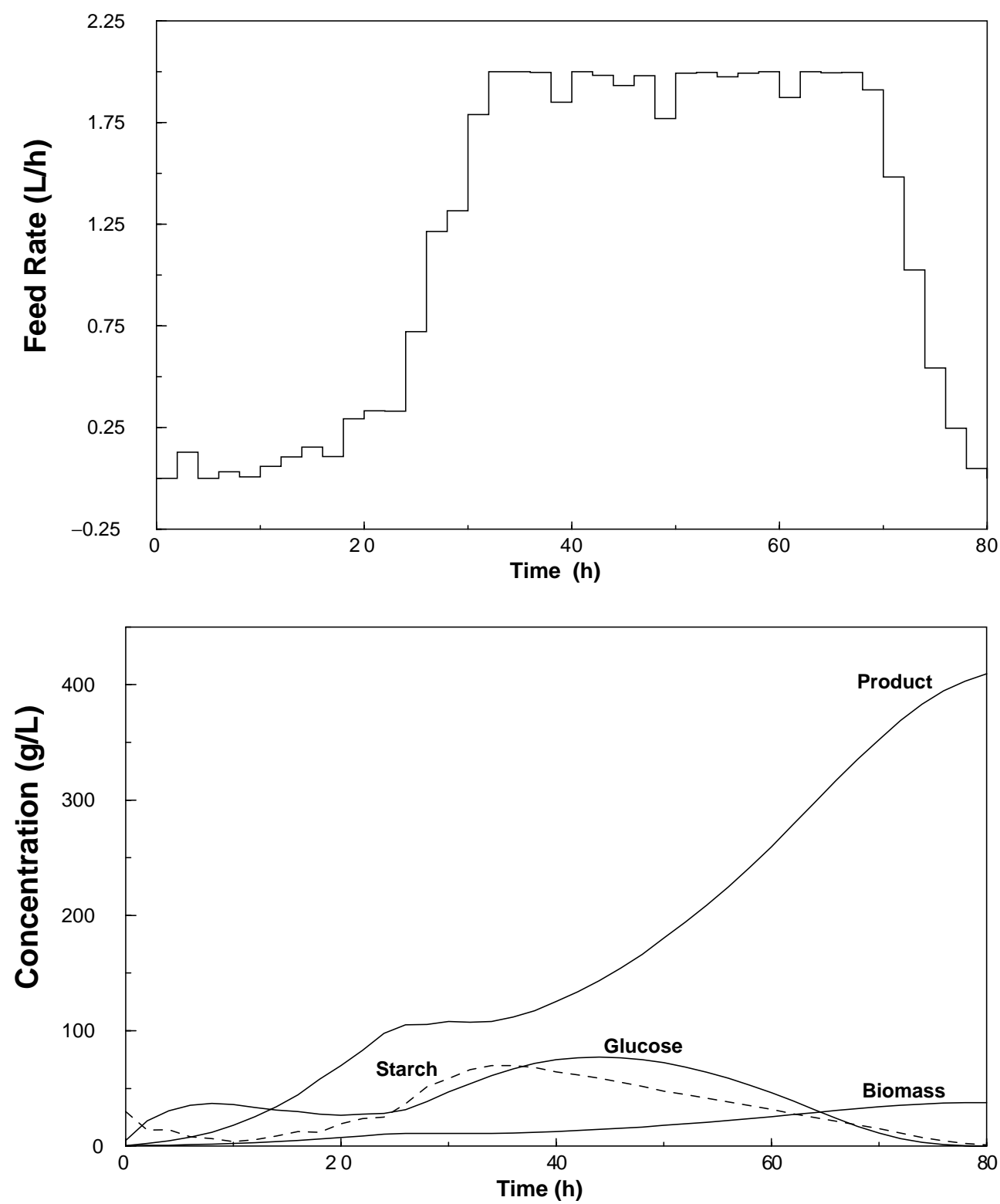

Fig. 5. Optimal feed flow rates and optimal concentration profiles for lactic acid production by simultaneous saccharification and fermentation.

parameter is determined as

$\tau_{\mathrm{Nu}}=t_{\mathrm{f}, \max }-\sum_{j=1}^{\mathrm{Nu}-1} \tau_{j}$

In the above step of optimization, it is possible that the summation of $\tau_{j}$ could get larger than $t_{\mathrm{f}, \max }$. In such a case, the (Nu)th time interval length $\tau_{\mathrm{Nu}}$ is set to zero. The successive steps of evolution during the progress of the algorithm will force the summation to be $\leq t_{\mathrm{f} \text {, max }}$ via constraint (15) and $\geq t_{\mathrm{f} \text {, min }}$ via constraint (16).

The Differential Evolution algorithm with the proposed method is used to solve this optimization problem with 79 decision variables (40 constant feed flow rates and 39 time interval parameters). The population size, $N_{\mathrm{p}}=150$ and maximum iterations of 20,000 are used to obtained the minimum solution for augmented problem. All other setting parameters and initial conditions are set to be same as mentioned in earlier section. The comparison of the results obtained using different time interval size parameters (i.e. non-uniform control vector parameterization) with those obtained with equal time intervals (i.e. uniform control vector parameterization) is shown in Table 3 . It is seen from Table 3 that higher final lactic acid concentration is obtained with higher production rate due to reduction in fermentation time. It is also clear from Table 3 that, the end constraints on substrate (glucose and starch) concentrations are met. The characteristics nature of optimal feed flow profiles and optimal concentration profiles obtained by the proposed method for $t_{\mathrm{f}}=72.48 \mathrm{~h}$ is same as those obtained with uniform control 
Table 3

Comparison of results obtained by optimal time intervals and equal time intervals for lactic acid production by SSF

\begin{tabular}{lccc}
\hline Parameters & \multicolumn{2}{l}{$\begin{array}{l}\text { Results with optimal } \\
\text { time intervals }\end{array}$} & $\begin{array}{l}\text { Results with equal } \\
\text { time intervals }\end{array}$ \\
\cline { 2 - 3 } & $t_{\mathrm{f}}$ changing & $t_{\mathrm{f}}$ fixed & \\
\hline$t_{\mathrm{f}}$ & 72.48 & 80 & 80 \\
$P\left(t_{\mathrm{f}}\right)$ & 411.02 & 418.15 & 409.45 \\
$P\left(t_{\mathrm{f}}\right) V\left(t_{\mathrm{f}}\right) / t_{\mathrm{f}}$ & 554.155 & 522.70 & 511.73 \\
$S\left(t_{\mathrm{f}}\right)$ & 1.023 & 1.02 & 0.97 \\
$G\left(t_{\mathrm{f}}\right)$ & 0.029 & 0.02 & 0.001 \\
\hline
\end{tabular}

vector parameterization (Fig. 5). Hence, use of non-uniform control vector parameterization and end-point constraints in Differential Evolution algorithm gives superior results in terms of end product concentration and production rate.

\subsection{Optimization studies of SSF process with multiple feeds}

The hydrolysis of starch as a feed could be rate limiting for higher productivity. It may therefore be useful to explore

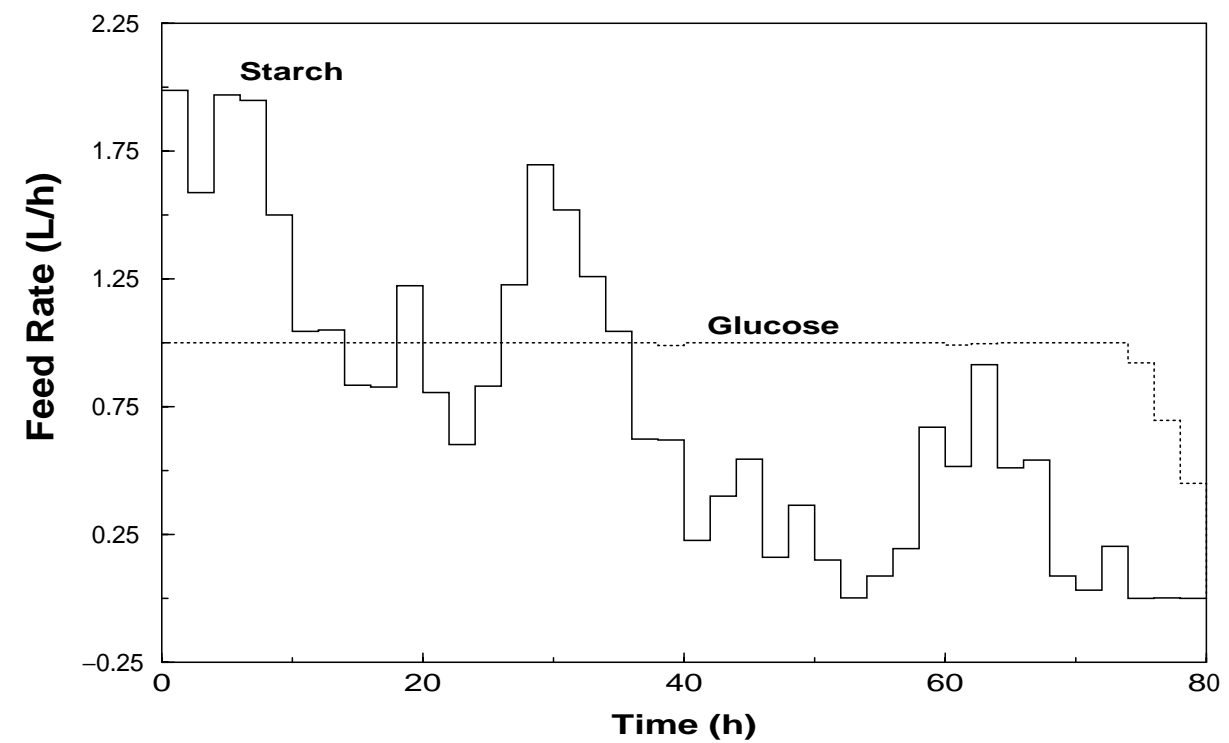

Fig. 6. Optimal feed flow rates for lactic acid production by simultaneous saccharification and fermentation with multiple feeds.

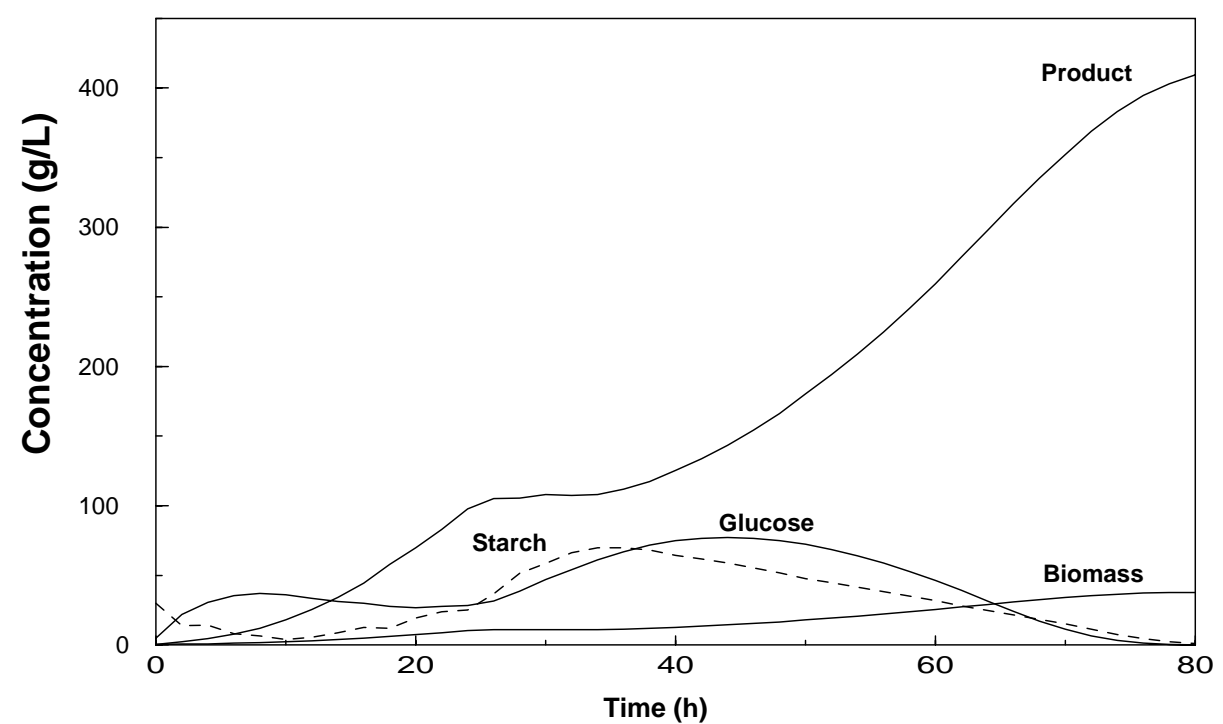

Fig. 7. Optimal concentration profiles for lactic acid production by simultaneous saccharification and fermentation with multiple feeds. 
minimal level of glucose addition at points where the starch hydrolysis is limiting. The other alternate of having high starch concentration is not feasible, as it inhibits the hydrolysis. This fact has provided an interest to follow the approach based on two separate feeds of starch and glucose instead of a single feed. If $F_{\mathrm{s}}$ and $F_{\mathrm{g}}$ are taken as the feeds which contain pure starch and pure glucose, respectively, then the dynamic mass balance equations for SSF process [12] can be written as

$\frac{\mathrm{d} X}{\mathrm{~d} t}=-\left(\frac{F_{\mathrm{g}}+F_{\mathrm{s}}}{V}\right) X+\mu X$

$\frac{\mathrm{d} S}{\mathrm{~d} t}=\frac{F_{\mathrm{s}}}{V} S_{\mathrm{F}}-\left(\frac{F_{\mathrm{g}}+F_{\mathrm{s}}}{V}\right) S-\frac{R_{\mathrm{G}}}{1.11}$ $\frac{\mathrm{d} G}{\mathrm{~d} t}=\frac{F_{\mathrm{g}}}{V} G_{\mathrm{F}}-\left(\frac{F_{\mathrm{g}}+F_{\mathrm{s}}}{V}\right) G+\left[R_{\mathrm{G}}-(a \mu X+b X)\right]$

$\frac{\mathrm{d} P}{\mathrm{~d} t}=-\left(\frac{F_{\mathrm{g}}+F_{\mathrm{s}}}{V}\right) P+(\alpha \mu X+\beta X)$

$\frac{\mathrm{d} V}{\mathrm{~d} t}=F_{\mathrm{g}}+F_{\mathrm{s}}$

where $G_{\mathrm{F}}$ and $S_{\mathrm{F}}$ are concentration of glucose and starch in $F_{\mathrm{g}}$ and $F_{\mathrm{s}}$, respectively. The kinetic model of the specific growth rate $\mu$ and the rate of saccharification $R_{\mathrm{G}}$ are taken to be the same as given in Eqs. (29) and (30).

As it has been clearly shown by Roy et al. [12] that, a combination of high concentration of glucose feed and a
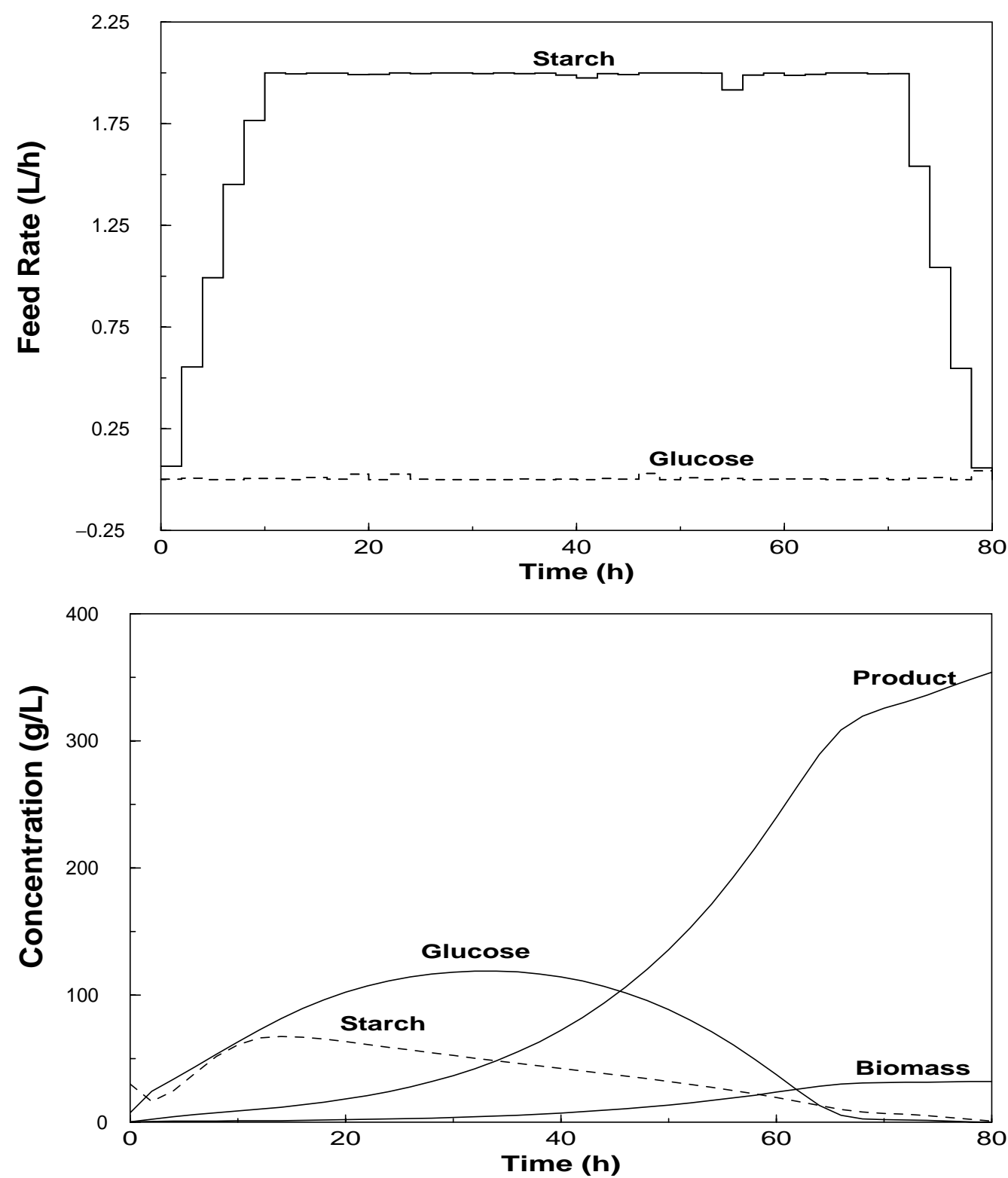

Fig. 8. Optimal feed flow rates and optimal concentration profiles for lactic acid production by SSF with penalized glucose addition when $S_{\mathrm{F}}=200 \mathrm{~g} / \mathrm{l}$. 
low concentration of starch feed with low initial concentration of starch, proves better in terms of fed-batch performance, the optimization problem is solved with $S_{\mathrm{F}}=50 \mathrm{~g} / \mathrm{l}$ and $G_{\mathrm{F}}=200 \mathrm{~g} / \mathrm{l}$. The initial concentration of glucose and starch are taken as 7.5 and $30 \mathrm{~g} / \mathrm{l}$, respectively. The initial and final working volume are 9 and 1501 . The residual limits on starch and glucose concentration are set equal to 1.0 and $0.1 \mathrm{~g} / \mathrm{l}$, respectively. The fermentation time is kept fixed as $80 \mathrm{~h}$ and initial biomass concentration is taken as $0.5 \mathrm{~g} / \mathrm{l}$. The upper limits on addition of glucose and starch sources are 1.0 and $2.0 \mathrm{l} / \mathrm{h}$, respectively. This optimization problem with 80 decision parameters (40 piecewise constant flow rates for starch and glucose feed each) and 83 inequality constraints is solved using proposed method with population size, $N_{\mathrm{p}}=150$. All other setting parameters are set to be same as discussed in earlier mentioned.

The maximum lactic acid concentration of $204.46 \mathrm{~g} / \mathrm{l}$ which is equivalent to production rate of $383.35 \mathrm{~g} / \mathrm{h}$ is obtained after 15,000 iterations. The concentration of starch and glucose at the end of SSF process are 0.21 and $0.1 \mathrm{~g} / \mathrm{l}$, respectively. The sum of all constraint violation equal to 0.013 means solution is nearly in feasible solution space. The optimal feed flow rates and optimal concentration profiles for biomass, starch, glucose and lactic acid are shown in Figs. 6 and 7, respectively. From the results obtained by proposed method, it is clear that during most of the fermentation time, the system predicts glucose flow rate at the upper bound of solution space which helps to keep the biomass growth rate high in spite of low saccharification rate. As a result the fermentation rate goes up. Also, the dilution effect caused by the addition of high volume, low concentration of starch keeps the glucose inhibition rate minimum over the whole time interval. As a result the starch concentration in the reactor never goes up, but decreases in a gradual manner. It is also clear from the Fig. 7 that, end-point constraints on substrate concentrations are met, i.e. the separation cost of lactic acid would be low in downstream processing.

\subsubsection{Computational results with penalty on glucose addition}

As glucose is an expensive source, its use increases the raw material cost for lactic acid production. On the other hand, starch is a relatively cheaper carbon source that is abundantly available and inexpensive. This fact has developed an interest to penalize the addition of glucose. The penalty on glucose addition is taken into account by adding the following constraint into the main optimization problem.

$$
\left(\sum_{j=1}^{\mathrm{Nu}} F_{\mathrm{g}}(j)\right) \times \frac{1}{\mathrm{Nu}}-G_{\text {addlimit }} \leq 0
$$

where $G_{\text {addlimit }}$ is the limit on the addition of glucose in each time interval if all time intervals are taken together. This is something which is different from the upper bound on the addition of glucose source. Though, $G_{\text {addlimit }}$ has to be less than upper bound of glucose feed rate, it allows the system to adjust the addition of glucose source in such a way that total glucose addition is less than $\mathrm{Nu} \times G_{\text {addlimit }}$.

This optimization problem with penalty on the addition of glucose source is solved using the Differential Evolution algorithm including Lagrange like method with the dynamic penalty function. The concentration of starch in $F_{\mathrm{s}}$ and that of glucose in $F_{\mathrm{S}}$ are taken same as $200 \mathrm{~g} / \mathrm{l}$. For simplicity the $G_{\text {addlimit }}$ is taken as $0.05 \mathrm{l} / \mathrm{h}$ which will approximate almost no addition of glucose. All the other setting parameters and initial conditions are set to be same as discussed in earlier section. The optimal feed flow rates and optimal concentration profiles for biomass, starch, glucose and lactic acid for this case are shown in Fig. 8. The maximum lactic acid concentration of $353.826 \mathrm{~g} / \mathrm{l}$ which is equivalent to production rate of $663.4 \mathrm{~g} / \mathrm{h}$ is obtained after 20,000 iterations. The sum of all flow rates of glucose feed is equal to 1.21 , which is less than $2.01(0.05 \times 401)$. It indicates that the path constraint on the addition of substrate source can be easily accommodated in Differential Evolution algorithm to give desired results.

\section{Conclusion}

The Differential Evolution with augmented Lagrangian including the dynamic penalty method was applied to fed-batch fermentation processes with state inequality constraints and bounds on feed rates. The concept of control vector parameterization (uniform/non-uniform) was used to transform the infinite dimensional optimization problems into a finite dimensional problems. The integration of the penalty functions for dynamic optimization problems was used to ensure that solutions lay within feasible domain. The proposed method was applied to decide optimal control policy in fed-batch fermentation for ethanol production and superior results were obtained using non-uniform control vector parameterization. The dynamic optimization of simultaneous saccharification and fermentation of starch to lactic acid was carried out using the proposed method. The performance of simultaneous saccharification and fermentation process was improved substantially in terms of end lactic acid concentration and productivity for single as well as multiple feed case. The main advantage of Differential Evolution with the augmented Lagrangian including dynamic penalty methods for fed-batch fermentation optimization is that substrate feed rate can be used as a control variable though there are several state inequality/equality constraints along with the bounds on the feed rate. This method is also a superior alternative to decide optimal feed policy for fed-batch fermentation with multiple feeds and path constraints on state variables to avoid inhibition. It is interesting to note that, the computational difficulties usually faced when dealing with constrained singular control problems were not encountered. Thus, it really provides a path to obtain the feasible optimal environment in fermentation broth. 


\section{Acknowledgements}

The first author gratefully acknowledges the funds provided as a scholarship by General Electric-India Technology Center under the "GE Fund Scholarship" scheme.

\section{Appendix A. Nomenclature}

$a$

parameter for growth associated glucose consumption $(\mathrm{g} / \mathrm{g})$

$b \quad$ parameter for non growth associated glucose consumption $(\mathrm{g} / \mathrm{g})$

$C_{\mathrm{R}} \quad$ crossover factor in Differential Evolution

$F \quad$ feed rate $(1 / \mathrm{h})$

$F_{\mathrm{m}} \quad$ mutation factor in Differential Evolution

$G \quad$ glucose concentration (g/l)

$J$ performance index in optimal control problem

$K_{\mathrm{G}} \quad$ glucose inhibition constant for saccharification (g/l)

$K_{\mathrm{I}} \quad$ glucose inhibition constant for fermentation (g/l)

$K_{1} \quad$ lactate inhibition constant for fermentation (g/l)

$K_{\mathrm{m}} \quad$ Michaelis-Menten constant (g/l)

$K_{\mathrm{S}} \quad$ glucose saturation constant $(\mathrm{g} / \mathrm{l})$

$N_{\mathrm{p}} \quad$ population size in Differential Evolution

$\mathrm{Nu}$ number of time partitions

$P \quad$ product concentration $(\mathrm{g} / \mathrm{l})$

$S \quad$ starch concentration $(\mathrm{g} / \mathrm{l})$

$t \quad$ time

$V \quad$ fermenter volume (1)

$V_{\mathrm{m}} \quad$ maximum rate of saccharification $(\mathrm{g} /(\mathrm{lh}))$

$\underline{x} \quad$ vector of state variables

$X \quad$ cell mass concentration $(\mathrm{g} / \mathrm{l})$

$\underline{u} \quad$ vector of control variables

\section{Greek letters}

$\alpha \quad$ parameter for growth associated lactate production $(\mathrm{g} / \mathrm{g})$

$\beta \quad$ parameter for non growth associated lactate production $(\mathrm{g} / \mathrm{g})$

$\mu_{\mathrm{m}} \quad$ maximum specific cell growth rate $(1 / \mathrm{h})$

$\phi \quad$ vector of system parameters

$\bar{\tau} \quad$ time interval parameter

\begin{tabular}{ll}
\multicolumn{2}{l}{ Subscripts } \\
$\mathrm{F}$ & feed \\
$\mathrm{f}$ & final \\
max & maximum
\end{tabular}

min minimum

r residual

0 initial

\section{References}

[1] Anuradha R, Suresh AK, Venkatesh KV. Simultaneous saccharification and fermentation of starch to lactic acid. Process Biochem 1999;35:367-75.

[2] Babu BV, Sastry KKN. Estimation of heat transfer parameters in a trickle-bed reactors using differential evolution and orthogonal collocation. Comput Chem Eng 1999;23:327-39.

[3] Biegler LT. Solution of dynamic optimization problems by successive quadratic programming and orthogonal collocation. Comput Chem Eng 1984;8:243-7.

[4] Chiou JP, Wang FS. Hybrid method of evolutionary algorithms for static and dynamic optimization problems with application to a fed-batch fermentation process. Comput Chem Eng 1999;23:127791.

[5] Diwekar UM. Batch distillation-simulation, optimal design and control. Washington: Taylor \& Francis; 1995.

[6] Lee MH, Han C, Chang KS. Dynamic optimization of a continuous polymer reactor using a modified differential evolution. Ind Eng Chem Res 1999;38:4825-31.

[7] Michalewicz Z, Schoenauer M. Evolutionary algorithms for constrained parameter optimization problems. Evol Comput J 1996;4:132.

[8] Modak JM, Lim HC. Simple nonsingular control approach to fed-batch fermentation optimization. Biotechnol Bioeng 1989;33:115.

[9] Modak JM, Lim HC, Tayeb YJ. General characteristics of optimal feed rate profiles for various fed-batch fermentation processes. Biotechnol Bioeng 1986;28:1396-407.

[10] Price KV. Differential evolution vs. functions of the 2nd ICEC. In: Proceedings of the IEEE Conference on Evolutionary Computations, Indianpolis, IN; 1997. p. 153-7.

[11] Ray WH. Advanced process control. New York: McGraw-Hill; 1981.

[12] Roy S, Gudi RD, Venkatesh KV, Shah SS. Optimal control strategies for simultaneous saccharification and fermentation of starch. Process Biochem 2001;36:713-22.

[13] Storn R. On the usage of differential evolution for function optimization. Berkeley: NAFIPS; 1996. p. 519-23.

[14] Storn R, Price K. Minimizing the real functions of the ICEC'96 contest by differential evolution. In: Proceedings of the IEEE Conference on Evolutionary Computation, Nagoya; 1996. p. 842-4.

[15] Storn R, Price K. Differential Evolution-a simple and efficient heuristic for global optimization over continuous spaces. J Global Optim 1997;11:341-59.

[16] Wang FS, Shyu CH. Optimal feed policy for fed-batch fermentation of ethanol production by Zymomous mobilis. Bioprocess Eng 1997; 17:63-8.

[17] Wang FS, Su TL, Jang HJ. Hybrid differential evolution for problems of kinetic parameter estimation and dynamic optimization of an ethanol fermentation process. Ind Eng Chem Res 2001;40:2876-85. 